The Egyptian Journal of Hospital Medicine (October 2018) Vol. 73 (10), Page 7726-7729

\title{
Massive Transfusion Complications Following Cutaneous Hemangioma
}

\author{
Rafeef Alhajress $^{1}$, Bader Muaykil AlQahtani ${ }^{2}$, Dhabiah AlQahtani ${ }^{3}$, Jaber Alenazi ${ }^{4}$ \\ 1- King Saud Bin Abdulaziz University for Health Sciences, Riyadh, 2- King Abdullah \\ Specialized Children's Hospital, National Guard Health Affairs, Riyadh, 3- King Saud Bin \\ Abdulaziz University For Health Sciences, Riyadh, 4- King Abdullah Bin Abdulaziz University \\ Hospital.
}

\begin{abstract}
Aim of the work: Describe the clinical events of a patient experiencing MT complications following cutaneous hemangioma resection since there is still no clear evidence on how to approach pediatric transfusion as the existing literature is lacking.

Methodology: Patient information was obtained from medical records after taking permission. Personal information that has been collected shall not be disclosed prior to consent. Patient's clinical course was mentioned in a chronological order with literature review to show significance of such cases in medical practice.

Results: A seven-month-old male who was found to have cutaneous hemangioma that required resection. Along to the clinical course, experienced several complications secondary to a massive transfusion.

Conclusion: Massive transfusion management in patients with non-traumatic-related hemorrhage, or surgery-related hemorrhage, is crucial for survival and better outcomes.
\end{abstract}

Keywords: transfusion, pediatric, hemangioma.

\section{Introduction}

The transfusion of blood and blood products is commonly used in a variety of clinical situations in an attempt to restore normal blood hemostasis and ensure adequate oxygen delivery. Massive transfusion (MT) involves the rapid administration of balanced ratios of packed red blood cells (RBCs), fresh frozen plasma (FFP) and Platelets (PLT) in significant volumes within a short time period; this practice is used to resuscitate critical patients and the methods involved are referred to as MT protocols (MTPs) ${ }^{[1,2]}$. While ,many definitions of MT have been established for the adult population, these are not, however, applicable for pediatric patients. The optimal approach to manage pediatric transfusion has not been adequately explored in the existing literature, leading to the practice of pediatric transfusion becoming reliant upon expert opinion and local institutional resources ${ }^{[1]}$.In general, the transfusion of blood and blood products has been associated with acute complications, which can manifest immediately, within minutes or up to 24 hours after transfusion. In fact, some cases experience delayed complications that may take days or even years to develop ${ }^{[3]}$. Acute complications can include fever, allergic reactions, anaphylaxis, acute hemolysis, blood-borne infections, transfusionassociated circulatory overload and transfusion-related acute lung injury. Delayed complications that are related to transfusion can include iron overload, posttransfusion purpura, transfusion-associated graft-versus-host disease and transfusionrelated immunomodulation ${ }^{[3,4]}$.The first case of pediatric MT to be reported in the literature involved a male neonate diagnosed with $\mathrm{Rh}$ hemolytic disease of the newborn (HDN). This case was managed with $400 \mathrm{ml}$ of blood in less than 24 hours and had an uneventful recovery thereafter ${ }^{[5]}$.

Aim of the work: In the present case report we described the clinical events of a patient experiencing MT complications following cutaneous hemangioma resection. 
To the best of our knowledge, a case such as this has yet to be reported in the literature.

\section{Methodology}

The patient was treated at King Abdullah Specialized Children Hospital in Riyadh, Saudi Arabia. Patient information were obtained from medical records after taking permission. Personal information that has been collected shall not be disclosed prior to consent. Patient's clinical course was mentioned in a chronological order. Literature review based on clinical findings was done, to emphasize the significance of recognizing such case in medical practice.

\section{Case Report}

Here, we reported the case of a sevenmonth-old male. He was born at full term at 37 weeks and was delivered by emergency caesarean section due to cephalopelvic disproportion (CPD). Measurements at birth were: weight $2.99 \mathrm{~kg}$, length $51 \mathrm{~cm}$ and head circumference $33.5 \mathrm{~cm}$. His APGAR score was 9,10 and 10 at 1,5 and 15 minutes, respectively. The mother was healthy and 20 years old who; she was not known to have significant medical history. Following delivery, the child was observed to have a large mass on the left thigh. The mass was bluish in some areas and measured $12 \times 23 \mathrm{~cm}$, extending from the femoral area to the knee. The mass was firm, tender and pulsating.

The patient subsequently underwent computed tomography (CT), which revealed a hemangioma with a feeding branch from the femoral artery and a draining vein to the femoral vein. Magnetic resonance imaging (MRI) was also performed, which showed a vascular soft tissue mass lesion with the possibility of a large muscular cavernous hemangioma. During his initial clinical course, the patient received packed RBCs, FFP and PLT several times due to ongoing bleeding within the mass, thus causing the mass to increase in size. The patient also commenced propranalol and predinsolone in an effort to manage the hemangioma and the primary team recommended that the patient should be transferred for vascular surgery in a specialized center.

At the age of seven months, the patient was admitted under our surgery team to excise the hemangioma. During surgery, the patient was suffered from massive bleeding that led to hemorrhagic shock and Disseminated Intravascular Coagulation (DIC). The child became hypotensive and turned pale intra-operatively because he suffered three episodes of bleeding. His estimated blood loss was in excess of $500 \mathrm{ml}$. Immediate resuscitation was performed with normal saline (NS) and packed RBCs. The surgeon opened the wound and found huge amount of blood came out with blood oozing throughout fascia and muscle surfaces, then he cauterized the source of bleeding, applied bleeding powder, re-inserted the drain, and the skin was closed with stapler, but 30 minutes later, the child bled again. The interventional radiologist was then involved for embolization. Post-surgery, the patient's laboratory analyses were as follows: Hemoglobin $=8.80, \quad \mathrm{PLT}=60, \mathrm{PT}=55$, $\mathrm{APTT}=165, \mathrm{INR}=5.76$ and $\mathrm{WBC}=15$. The patient underwent aggressive transfusion with 2 units of packed RBCs and PLT (100 $\mathrm{ml}$ every 4 hours $), 2$ units of FFP $(100 \mathrm{ml}$ every 4 hours) and Factor VII (200 mg every 2 hours for a total of 5 doses). After stabilization, the child was transferred to the ward for recovery and observation. On the ward, the child was treated with continued ventilation and oxygen saturation was maintained in excess of $95 \%$. The child was also given fentanyl $0.001 \mathrm{mg} / \mathrm{kg} / \mathrm{hour}$, midazolam $\quad 0.001 \mathrm{mg} \quad / \mathrm{kg} /$ minute, vancomycin $10 \mathrm{mg} / \mathrm{kg}$ every 6 hours and ceftriaxone $50 \mathrm{mg} / \mathrm{kg}$ once daily. He received 3 units of PLT, packed RBCs ( $250 \mathrm{ml}$ over 2 hours), one unit of FFP and platelets every 6 hours (100 $\mathrm{ml}$ on each occasion). In addition, the child received $5 \mathrm{ml}$ of calcium gluconate over 30 minutes and 200 mic of Factor VII every 2 hours for a total of 4 doses.

On the third day after admission, the child had two seizure attacks with hypoglycemia; he also suffered edema and 
increased thickness of the chest wall. Moreover, nasogastric tube (NGT) showed coffee ground aspirate. As a result of these sudden events, appropriate and prompt management was initiated including midazolam for seizures, dextrose $10 \%$ in water, MRI and neurology consultation. We also commenced furosamide and ranitidine for edema and gastrointestinal problems.

On the fourth day, the child developed red man syndrome while on vancomycin level $131 \mathrm{ug} / \mathrm{ml}$. He looked clinically edematous and was hypotensive with cold extremities. Further examination revealed that the anterior fontanel was full, tense and bulging. He also had a distended abdomen and had lost $50 \mathrm{ml}$ of blood over the last 7 hours.

After all these unfavorable incidents, our plan was to maintain vancomycin until level decreased to $20 \mathrm{ug} / \mathrm{ml}$, administer $20 \mathrm{ml}$ of NS, infuse $0.002 \mathrm{mg} / \mathrm{kg} /$ minute of epinephrine, administer $15 \mathrm{mg}$ of hydrocortisone, discontinue midazolam, continue furosamide for hypervolemia and keep transfusion of 1 unit of FFP and 1 unit of PLT. The child was then transferred to the Pediatric Intensive Care Unit (PICU) where he subsequently was suffered from generalized edema, respiratory acidosis with increased base deficit and his PLTs fell to 66 . The child received intensive management in the PICU and was intubated and placed on a ventilator; a chest X-ray was carried out to investigate for possible pulmonary edema. $\mathrm{He}$ was administered with metalozone, chloral hydrate, hydralazine and laxative lactulose. Ceftrixone was changed to meropenum. Hydrocortisone and multivitamins were stopped. Also, albumin was given $50 \mathrm{ml}$ over 2 hours due to hypoalbuminemia. On the next morning the patient arrested, CPR done for 5 minutes, and 1 dose of epinephrine was given.

After stabilization, an MRI was requested, based on clinical findings, which showed some ischemic changes in the corpus callosum. He was extubated and given dexamethasone nebulization immediately after. There was no edema or complications noted after extubation. Phenobarbital was given to control his seizures. $\mathrm{He}$ was discharged after insuring that he was clinically stable and his vitals and labs were within normal limit. Arranged follow up visits in clinic.

\section{Discussion}

Complications arising from the surgical resection of a hemangioma may vary from mild to catastrophic consequences. The most common and devastating complication is a massive hemorrhage which may result in multi-organ failure and death, complicated by secondary infection manifesting as an erythematous and crusting skin ulceration around the source of bleeding ${ }^{[6]}$. Several reported cases which highlight the most common complications associated with hemangioma, included infection, necrosis, scarring, and compressive complications such as upper airway occlusion or eye involvement causing amblyopia ${ }^{[5-7]}$.One paper focused particularly on hematological complications and reported that, of the cases examined, $76 \%$ (13 cases out of 17) developed thrombocytopenia with a platelet count reference range of 7 to $119 \times 10^{3} / \mu \mathrm{L}^{8}$. Mortality rate among 17 cases was $30 \%$ despite medical, surgical, and embolization intervention ${ }^{[8]}$. While, several definitions of MT have been established for adults, the situation for pediatrics is much more complicated. Total body volume (TBV) in pediatrics varies among different age groups making the management of severe bleeding in children largely dependent upon age and weight. Neonatal transfusions depend mostly on expert opinion and local resources rather than high quality published data ${ }^{4}$. In pediatrics, MT is defined as an RBC transfusion of $50 \%$ of the TBV over 3 hours, $100 \%$ of the TBV over 24 hours, or $>10 \%$ of the TBV per minute ${ }^{1}$. RBC transfusion is indicated for massive blood loss which results in $>15 \%$ reduction of $\mathrm{TBV}$ due to surgical complications or trauma. Other indicators for anemia include hemoglobin of $\leq 8 \mathrm{~g} / \mathrm{dL}$ in patients undergoing emergent 
surgery, or experiencing symptoms of anemia. Indications for PLT transfusion include a PLT count $<50,000 / \mu \mathrm{L}$ with active bleeding or undergoing an invasive procedure. Platelet dysfunction of any cause (acquired or inherited) is a clear indication of PLT transfusion. Both cryoprecipitate and FFP can be used in cases of disseminated intravascular coagulation (DIC) and in massive transfusion situations ${ }^{9}$.

In our present case, we experienced a massive transfusion-related circulatory overload with lung damage. The existing literature indicated that long-term complications mostly involve delayed hemolytic reactions, iron overload, microchimerism, post-transfusion purpura, transfusion- related graft-versus-host disease, and transfusion-related immunological complications ${ }^{2}$. Furthermore, there are other, less common, complications associated with MT, including electrolyte and metabolic disturbances, hypothermia arising from exposure to cold products, infections and transfusion-related necrotizing enterocolitis (NEC) ${ }^{[1,3]}$.It is clearly evident that massive transfusion management in patients with nontraumatic-related hemorrhage, or surgeryrelated hemorrhage, is crucial for survival and better outcomes. Our aim is to expand the knowledge regarding pediatric massive transfusion clinical course and to build solid evidence based practice in the future to treat patients requiring massive transfusion for better outcome.

\section{References:}

1. Diab YA, Wong ECC, Luban NLC (2013): Massive transfusion in children and neonates. BJH., 161(1):15-26.
2. Dehmer JJ, Adamson WT (2010): Massive transfusion and blood product use in the pediatric trauma patient. Seminars in pediatric surgery, 19(4):286-91.

3. Sharma S, Sharma P, Tyler LN (2011): Transfusion of blood and blood products: indications and complications. American family physician, 83(6):719-24.

4. Parker RI (2014): Transfusion in critically ill children: indications, risks, and challenges. Critical care medicine, 42(3):675-90.

5. Bloxsom A (1946): Hemolytic disease of the newborn (erythroblastosis fetalis): Treatment by a single massive transfusion, with complete recovery. American Journal of Diseases of Children, 72(3):320-4.

6. Beck DO, Gosain AK (2009): The presentation and management of hemangiomas. Plastic and reconstructive surgery, 123(6):181e-91e.

7. Agesta N, Boralevi F, Sarlangue J, Vergnes P, Grenier N, Leaute-Labreze C (2003): Life-threatening haemorrhage as a complication of a congenital haemangioma. Acta paediatrica, 92(10):1216-8.

8. Weitz NA, Lauren CT, Starc TJ, Kandel JJ, Bateman DA, Morel KD et al. (2013): Congenital cutaneous hemangioma causing cardiac failure: a case report and review of the literature. Pediatric dermatology, 30(6): e180-90.

9.https://www.bcw.edu/cs/groups/public/doc uments/documents/lwd1/awrl/ edisp/pediatr ic-transfusion-guidelin.pdf. 